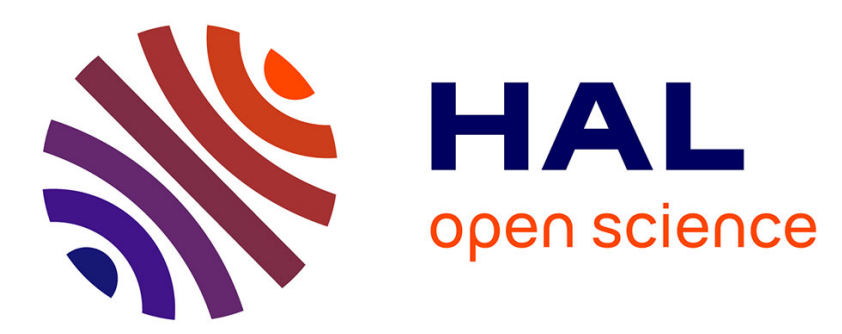

\title{
A spectral model for interchange transport in tokamak scrape-off layers
}

M. Peret, N. Fedorczak, P. Tamain, Ph Ghendrih, Ph. Ghendrih, L. Vermare

\section{To cite this version:}

M. Peret, N. Fedorczak, P. Tamain, Ph Ghendrih, Ph. Ghendrih, et al.. A spectral model for interchange transport in tokamak scrape-off layers. Nuclear Fusion, 2021, 61 (4), pp.046045. 10.1088/17414326/abe6b3 . hal-03440223

\section{HAL Id: hal-03440223 \\ https://hal.science/hal-03440223}

Submitted on 30 Nov 2021

HAL is a multi-disciplinary open access archive for the deposit and dissemination of scientific research documents, whether they are published or not. The documents may come from teaching and research institutions in France or abroad, or from public or private research centers.
L'archive ouverte pluridisciplinaire HAL, est destinée au dépôt et à la diffusion de documents scientifiques de niveau recherche, publiés ou non, émanant des établissements d'enseignement et de recherche français ou étrangers, des laboratoires publics ou privés. 


\title{
A spectral model for interchange transport in tokamak scrape-off layers
}

\author{
M. Peret ${ }^{1, *}$ N. Fedorczak ${ }^{1}$, P.Tamain ${ }^{1}$, Ph. Ghendrih $^{1}$, L. Vermare $^{2}$, Tore \\ Supra and WEST teams ${ }^{1}$ \\ ${ }^{1}$ CEA, IRFM, F-13108 Saint-Paul-Lez-Durance, France \\ ${ }^{2}$ LPP, F-91128 Palaiseau, France \\ * Corresponding author \\ E-mail: mathieu.peret@cea.fr
}

\begin{abstract}
Convection of plasma filaments (blobs) is commonly considered as responsible of the transport across flux surfaces in scrape off layer (SOL) of fusion devices. The turbulent simulations are able to reproduce the local behavior of this filamentary transport, i.e. structure sizes and velocities and the resulting SOL width in agreement with experimental observations in circular plasma. On the other hand, these simulations being time consuming and hard to interpret, reduced models are needed. In this reducing approach, the intermittency has not yet been described and keeps one from drawing a transport model. This key point motivated a switching of point of view in order to draw a predictive model for transport based on an analytical and spectral description proposed in this contribution. This model decomposes the radial flux into contributions from poloidal waves of which behavior is dictated by interchange mechanisms. A resulting prediction of density and electrostatic potential spectra leads to an expression of the time averaged radial flux. The SF model allows one to predict the turbulent structures behavior (amplitudes, velocities, sizes) but also fluctuation levels and macroscopic quantities such SOL width. This model is confronted with flux-driven simulations and experimental measurements showing a quantitative agreement between predictions and observations for the simulated turbulence behavior and both simulated and measured features, e.g. turbulence features, SOL width and fluctuation levels. Expressing the model in terms of engineering parameters, comparisons with scaling laws and extrapolations for future devices shows a good recovering.
\end{abstract}

Keywords: Scrape-off layer width, turbulent transport, spectral model, ITER predictions

Submitted to: unknown

\section{Introduction}

The issues of plasma-wall interaction and power exhaust are key questions for the quest for controlled fusion in magnetic fusion reactors. Indeed, the heat flux from the plasma core going through the edge and towards the target parts of the tokamak vessels is still not fully understood. This edge region is the framework of an intense radial transport leading the heat flux in the open field line zone of the plasma: the scrape-off layer (SOL). The SOL properties such as the heat flux decay length $\lambda_{q}$ is setting the amplitude of flux impacting the wall. The density decay length $\lambda$ will set the amplitude of the particle flux on the targets and will dictate phenomena as erosion sputtering and also trapping in the wall. The idea of intermittent filaments (blobs) drifting outwards and carrying this "anomalous" transport is commonly accepted. But so far, no theoretical model has shown an ability to predict these main SOL properties leading to the use of experimental scaling laws $[1,2]$. Numerical simulations are able to reproduce both the behavior of the intermittent turbulence and global features $[3,4,5]$ such as the SOL width adimensioned by Larmor radii $\left(\rho_{i}\right)$ in agreement with experimental measurements $[6,7]$, e.g. $100 \rho_{i}$ in inner-limited plasma [8] and $1-10 \rho_{L}$ in diverted configurations. 
The experimental characterization of the filaments is based mainly on the concept of "mean blobs" which is hard to define because one can only measure intermittent large events compared to the mean profiles. The point is that the intermittent approach keeps one from linking these isolated events with the average radial flux and so with the SOL width. A huge effort on simulating this transport has been carried during the last decades. On the other hand, the issues of time-costing and complexity of the simulations pointed out the need of reduced models for SOL transport. Despite the theoretical effort in order to describe filament properties $[9,10,11]$, only velocities and sizes of filaments are described but not their intermittent and amplitude features[12]. Another point of view draws SOL and transport properties from distribution functions describing filament properties [13] but these distribution functions are not predicted and usually ad-hoc. Finally, recent works $[5,14]$ have focused on a spectral analysis based on a single wavenumber representing the turbulence, with a collisional description of the parallel dynamics, leading to scaling of SOL width in qualitative agreement with measurements. Strikingly, the sheath end of the field lines is not considered in the model, thus questioning the validity of this SOL model.

This contribution proposes a self consistent spectral model of sheath limited interchange turbulence in circular geometries, as initiated in [6]. An analytical description of time averaged poloidal spectra of density and potential fluctuations is presented. These spectra can then be applied to predict both local and global transport properties like fluctuation levels and SOL width. In particular intermittency is simply smeared out by the time average nature of the model.

In the following, the set of conservation equations for $2 \mathrm{D}$ interchange turbulence is first introduced in section 2 . In section 3 , the spectral filament model ( $\mathrm{SF}$ model) is derived. The verification of the SF model is done in section4 by comparing its predictions against non linear simulations of the initial equations with TOKAM-2D. Then, a validation of the SF model is proposed in section 5 against an experimental database of different Langmuir probes measurements in circular and ohmic discharges of Tore Supra. Finally, a discussion is proposed in section 6 covering two important aspects of the model: the link with blob intermittency, and extension of the model to incorporate more complex physics like divertor geometry, collisions and ExB flow shearing.

\section{A 2D isothermal model for interchange transport}

In order to investigate the physics of interactions between filamentary transport and background plasma profiles in a self-consistent way, the use of flux-driven models is particularly indicated, such as in TOKAM-2D or HESEL [3, 15]. Although 3D simulation codes have started to produce data to compare with experiments $[16,17]$, cases are still spare and much more complex to interpret than simpler $2 \mathrm{D}$ simulations (transverse to magnetic field lines). In particular, 2D simulations have shown robust agreements against experimental observations [3, 6, 7]. The isothermal flux-driven TOKAM-2D code [3] is based on the continuity equation and on the total charge conservation, leading to density and vorticity conservation equations. It models the system as a competition between two phenomena. First, the curvature polarization drives the interchange instability. A constant parameter $g$ represents the mean effect of the curvature drive along a field line. In opposition to this contribution, the current going through the sheath at the plasma wall interface limits the growth of interchange instability. The sheath impact is modelled by an effective parallel loss rate

\section{$\sigma_{\|}$}

acting as a charge loss in the vorticity equation and a particle loss in the continuity equation:

$$
\begin{aligned}
\left(\partial_{t}-D \Delta\right) n & =[n, \Phi]-n \sigma_{\|} e^{\Lambda-\Phi}+S \\
\left(\partial_{t}-\nu \Delta\right) \Delta \Phi & =[\Delta \Phi, \Phi]+\sigma_{\|}\left(1-e^{\Lambda-\Phi}\right)-g \frac{\partial_{y} n}{n}
\end{aligned}
$$

Time is normalized to the ion cyclotron period $\Omega_{i}^{-1}=\frac{A m_{p}}{Z e B}$ and space to the ion Larmor radius $\rho_{i}=\sqrt{\frac{A m_{p}\left(T_{e}+Z T_{i}\right)}{Z e B^{2}}}$ where $A$ is the number of nucleons, $m_{p}$ is the proton mass in $\mathrm{kg}, B$ is the magnetic field amplitude in T, $Z$ is the ion charge number, $T_{e}$ and $T_{i}$ are respectively the electron and ion temperature in $\mathrm{eV}$ and $e$ is the elementary charge in C. Plasma potential is normalized to electron temperature $T_{e}$ and $\Lambda$ is the potential imposed by the sheath $(\approx 3)$. The two dimensions $x$ and $y$ are respectively the radial direction and the electron diamagnetic drift direction close to the poloidal direction and assumed to be transverse to the magnetic field. The expressions of the curvature and the parallel loss rate are given by $g=\alpha_{g} \frac{\rho_{i}}{R}$ and $\sigma_{\|}=\frac{\rho_{i}}{\pi q R}$ where $R$ is the plasma major Radius in meter, $q$ is the cylindrical safety factor and $\alpha_{g}$ is a constant taking into account the geometry, including the effective tilt 
of flux tubes by the magnetic shear. Convection by electrostatic drift is described by the Poisson bracket $[f, \Phi]=\partial_{x} f \partial_{y} \Phi-\partial_{y} f \partial_{x} \Phi . D$ and $\nu$ are respectively diffusion and viscosity coefficient normalized to the Bohm diffusivity $D_{B}=\rho_{i}^{2} \omega_{i}$. The system is forced by a particle source $S$ localised in the $x$ direction and usually uniform in the $y$ direction.

\section{Spectral Filament model (SF model)}

\subsection{Fundamental principle of the SF model}

The density SOL width $\lambda$ can be deduced by a simple balance between radial flux and the parallel flux going to the targets. The turbulent radial flux is assumed to be carried by filaments moving radially due to the curvature polarization. This motion is at least limited by parallel losses toward the target. In order to predict $\lambda$ we build a model of contributions of modes to the radial flux $\Gamma_{r}$. To subvert the issue of intermittent transport, this work aims at describing a time averaged radial flux. Assuming that the turbulent flux is responsible for the time averaged radial transport, one can express it as $\Gamma_{r}=<\tilde{V} \tilde{n}>_{t}$. If perturbations of potential and density are described as poloidal waves (of amplitude $f_{k}$ at the wave number $k$ ), potential and density can be locally written as $\Phi=\Lambda+\int_{-\infty}^{+\infty} \Phi_{k} \cos \left(k y+\psi_{k}\right) d k$ and $n=n_{0} e^{-x / \lambda}\left(1+\int_{-\infty}^{+\infty} n_{k} \cos (k y) d k\right)$, where $\psi_{k}$ the phase shift between perturbation of potential and density of the mode $k$.

The poloidal $(y)$ average of the radial particle flux can be deduced after a few straightforward steps: $\Gamma_{r}=8 \pi^{2} n_{0} e^{-x / \lambda} \int_{0}^{+\infty} k n_{k} \Phi_{k} e^{i\left(\frac{\pi}{2}-\psi_{k}\right)} d k$, taking into account the parity of the spectra. The flux balance in the SOL then leads to a relation between the radial gradient of the radial flux and the parallel losses: $\partial_{r} \Gamma_{r}=-\sigma_{\|} n_{0} e^{-x / \lambda}$. Assuming that the effective transport parameter $\Gamma_{r} / \bar{n}$ is radially constant, one can equal the decay length of the flux with the one of the density and obtain the following expression :

$$
\lambda=\frac{8 \pi^{2}}{\sigma_{\|}} \int_{0}^{+\infty} k n_{k} \Phi_{k} e^{i\left(\frac{\pi}{2}-\psi_{k}\right)} d k
$$

Looking to equation (3), it is obvious that the SF model needs to have only access to the knowledge of three spectra for mode amplitude $n_{k}$, velocity $k \Phi_{k}$ and the phase shift $\psi_{k}$. Furthermore, these spectra may depend on the SOL width themselves making equation (3) non-linear and self-consistent. This approach is valid at each time but the aim of the SF model is to develop a time average solution. To be able to make the calculations in this paradigm, we make an assumption by equaling the time average of the flux to the integral of the time averaged spectra. Although rough, this approximation is verified on non linear simulations and discussed at the end of the paper. Moreover, the expression of the phase shift $\psi_{k}$ is hard to draw but one can easily understand with equation (3) that the maximum flux is due to $\psi_{k}=\pi / 2$ which can be obtained by a linear solution of the system. Consequently, the model will consider a constant phase shift equal to $\pi / 2$, corresponding to the maximum coupling between density and potential perturbations. All these assumptions will be discussed later in this contribution.

\subsection{Spectra of potential and density}

3.2.1. Isolated filament model Isolated filament models are generally focused on predicting the drift velocity of isolated density perturbations [18]. To do so, the vorticity conservation equation is simplified by an ordering approach, balancing vorticity sources and sinks expressed with filament parameters: an inverse transverse size $k$ and a normalized density perturbation $n_{k}$ (filament density perturbation over background density):

$$
k^{4} \Phi_{k}^{2}+\sigma_{\|} \Phi_{k}=g k n_{k}
$$

The first left hand side term represents the limitation of the motion of the filament by its inertia according to [18]. The second one characterizes the limitation of the motion by the parallel losses. Both oppose the curvature term in the right-hand side of (4) driving the instability and each represents a regime (inertial or sheath limited) separated by the wavenumber $k_{0}=\left(\frac{\sigma_{\|}}{4 g n_{k}}\right)^{1 / 5}$

This ordering model for an isolated filament will simply be transposed to the spectral description. In principle, the non linear inertial term cannot be identically translated to spectral components due to non linear convolutions in the Fourier space. For now this is omitted in purpose, but will be refined in the following section. Resolving (4) gives a dependency of $\Phi_{k}$ function of the density spectrum $n_{k}$ and the wavenumber $k$ that we label as isolated filament (IF) model for the potential:

$$
\Phi_{k}=\frac{\sigma_{\|}}{2 k^{4}}\left[-1+\sqrt{1+\frac{k^{5}}{k_{0}^{5}}}\right]
$$

To complete the model, one needs to derive an expression of $n_{k}$. Equation (1) shows that the impact of the perturbations are taken into account into only two terms : the Poisson bracket representing the convection of the gradient of density and the parallel losses. Neglecting this last contribution, the system 
is reduced to the convection of the background gradient of density counter-balanced by the convection of the mode by itself, in other words it reduces to the common mixing length rule:

$$
n_{k}=\frac{1}{\lambda k}
$$

Fig.1 shows a comparison of the isolated filament model for the potential spectrum and the mixing length rule for the density spectrum, against spectra built from a non linear flux driven simulation of the initial equations, using TOKAM-2D code. The agreement is relatively good regarding both the shapes and amplitudes of the spectra, noting that: the isolated filament potential model overestimates the simulation spectrum for $k>k_{0}$, and the mixing length rule overestimates the simulation spectrum for $k<k_{0}$. If one looks at the contribution of the mode $k$ to the total radial flux, agreement is again quantitative except a moderate overestimation of the model for $k>k_{0}$. The phase shift between density and potential in the simulation is around $\pi / 3$ and not $\pi / 2$ as assumed, but this only changes the flux amplitude by $10 \%$.

Discrepancies between the isolated filament model and the simulation spectra suggest that nonlinearities are potentially not correctly treated in the current model, even though the impact seems quite moderate. A refinement of the isolated filament model is proposed in the following, taking into account an effective diffusivity due to the dynamics of the dominant mode $k_{0}$.

\subsubsection{Limitation of spectra by the dominant} mode $k_{0}$ The previous illustration of simulation spectra (fig.1) reveals an important feature of the interchange turbulence: a packet of modes around $k=k_{0}$ exhibits a much faster drift velocity than the whole remaining of the spectrum. The sound agreement of the isolated filament model regarding the position and amplitude of this maximum in the potential spectrum indicates that this dominant mode is the mode sitting at the transition between sheath limited and inertial regimes.

This mode $k_{0}$ drifts outward down the density gradient faster than all other modes. We can picture that while drifting, the mode will necessarily drift over modes of lower velocities. These modes will therefore experience a finite perturbation from the fast passing vortices of the dominant mode. The exact description of this perturbation is not the focus of this work: we seek for an ordering approach to stay in line with the paradigm of the isolated filament. First, the passing vortices of the dominant mode will act as a turbulent viscosity $\nu_{\text {eff }}=\Phi_{k_{0}}$, spreading the vorticity of all other modes. The model for potential spectra can therefore be rewritten adding this effective viscosity, but also removing the inertial term that is not correct in the Fourier space:

$$
k^{4} \Phi_{k} \Phi_{k_{0}}+\sigma_{\|} \Phi_{k}=g k n_{k}
$$

Interestingly the later equation is identical to the isolated filament model when applied to the dominant mode $k_{0}$ : the effective viscosity of the dominant mode acting on itself is equivalent, in effect, to the inertial damping mechanism. In other words, this new model for potential spectra conserves the agreement against simulations for the prediction of $\Phi_{k_{0}}$. This effective turbulent mixing from the dominant mode should, in principle, also act on the density field. That said, adding an effective diffusivity in the form $D_{e f f}=\Phi_{k_{0}}$ in the density model will result in a strong coupling between the two equations for density and potential spectra, that is uneasy to manipulate. To tackle this, an heuristic approximation is proposed. When dominant vortices $\left(k_{0}\right)$ drift over density modes of larger sizes $\left(k<k_{0}\right)$, the density pattern of these modes is disturbed by the strong vortices, as is the background density gradient in the mixing length paradigm. For these large size density modes, it represents a loss of matter. To stick with the mixing length rule, that did not show to be completely wrong against simulated density spectra, we will simply replace the wavenumber appearing in the later by an effective hybrid wavenumber:

$$
n_{k}=\frac{1}{\lambda \sqrt{k^{2}+k_{0}^{2}}}
$$

Such an approximation of the non linear impact of the dominant mode on the rest of the spectrum has the good advantage to keep the model simple, to provide a saturation at low $k$ and to lower the density spectrum at $k_{0}$, as required to gain in quantitative agreement against simulation results.

\subsubsection{The spectral filament model The new} equations for density and potential spectra $(7,8)$ are meant to include an approximation of the non linear coupling with the dominant mode $k_{0}$, that appears as a coupling between the density and potential equations through the wave number $k_{0}$. In order to derive two independent expressions for the density and potential spectra, a first step is needed: derive the expressions of $k_{0}$ and $\Phi_{k_{0}}$. The later comes from a straightforward evaluation of equation 7 at $k=k_{0}$, including the new expression of the density spectrum 8. The wavenumber $k_{0}$ is defined as the wavenumber where the potential spectrum reaches its maximum: $\left.\partial_{k} \Phi_{k}\right|_{k_{0}}=0$. Here, the expression of the potential 
spectrum $\Phi_{k}$ is given by 7 , where the density spectrum is replaced by its new expression 8 . Doing so, a new expression of $k_{0}$ is derived function of the three control parameters $g, \sigma_{\|}$and $\lambda$.

These two intermediate steps done, the whole system can be solved into analytic and independent expressions of the density and potential spectra. After some algebra, the spectral filament (SF) model leads to the following expression of density and potential spectra:

$$
\begin{aligned}
k_{0} & =\left(\frac{0.92 \lambda \sigma_{\|}^{2}}{4 g}\right)^{1 / 4} \\
u & =\frac{k}{k_{0}} \\
\Phi_{k} & =\frac{g}{\lambda \sigma_{\|}} \frac{u}{\sqrt{1+u^{2}}\left(1+0.14 u^{4}\right)} \\
n_{k} & =\frac{1}{\lambda k_{0}} \frac{1}{\sqrt{1+u^{2}}}
\end{aligned}
$$

In fig.1, we can see that the SF model is better describing the simulation spectra than the previous isolated filament model. Moreover it leads to easy integration of the spectra which is not the case of the isolated filament model as discussed hereafter.

\subsubsection{Contributions to the flux and SOL width}

With the system of eqs. $(11,12)$, the model gives access to the contribution $k \Phi_{k} n_{k}$ of each mode to the total radial turbulent flux.

$k \Phi_{k} n_{k}=\frac{\sigma_{\|} k_{0}^{2}}{2 \lambda} \frac{1}{u^{3} \sqrt{u^{2}+1}}\left[-1+\sqrt{1+\sqrt{2} \frac{u^{5}}{\sqrt{u^{2}+1}}}\right]$

This function has no straightforward analytical integral expression, but it can be expressed as a product of two independent functions: a scalar containing the physical amplitude of the flux, and a spectral shape function of the normalized wavenumber only. The later can be integrated numerically to retrieve an analytical expression of the total turbulent flux function of $g, \sigma_{\|}$and $\lambda$. Similarly, the expressions of density and potential spectra can be integrated with the same manner to express the fluctuation levels of these quantities: $\sigma_{f}=\sqrt{8 \pi^{2} \int_{0}^{+\infty} f_{k}^{2} d k}$.

After some algebra, the followig expressions are derived for the radial particle flux, the density and potential fluctuation levels, and the SOL width $\lambda$ :

$$
\begin{aligned}
\Gamma_{r} & =\bar{n} 41 \lambda^{-7 / 4} g^{3 / 4} \sigma_{\|}^{-1 / 2}\left[c_{S}\right] \\
\frac{\sigma_{n}}{\bar{n}} & =13.3 \lambda^{-9 / 8} g^{1 / 8} \sigma_{\|}^{-1 / 4} \\
\frac{\sigma_{\Phi}}{T_{e}} & =5.0 \lambda^{-7 / 8} g^{7 / 8} \sigma_{\|}^{-3 / 4} \\
\lambda & =3.9 g^{3 / 11} \sigma_{\|}^{-6 / 11}\left[\rho_{L}\right]
\end{aligned}
$$

\section{Verification of the SF model against simulations}

A set of 8 non linear flux driven simulations of equations $(1,2)$ is built with the TOKAM2D code. The simulation domain is set as $N_{x} \times N_{y}=512 \times 256$ with the $y$ direction periodic and $x$ direction limited by boundary buffers. The source term is $y$-uniform and located in the low $\mathrm{x}$ region. Diffusion and viscosity are set to $D=\nu=1 \times 10-4$, small enough to neglect their effects on the interchange turbulence [7]. Simulations are run up to statistical steady states, over which fluctuations and profiles properties are extracted. To avoid any boundary effects from source and buffers, quantities are measured in a central simulation domain $(x \in[50-200])$. Curvature $g$ and parallel loss rates $\sigma_{\|}$are scanned over experimentally relevant ranges of cylindrical safety factor and normalized Larmor radius $\left(g \in[1,40] \cdot 10^{-4}\right.$ and $\left.\sigma_{\|} \in[1,50] \cdot 10^{-5}\right)$.

The primary purpose of SF model is to predict the the time averaged poloidal spectra of density and potential fluctuations. A detailed comparison against a single simulation is shown in fig. 1 .

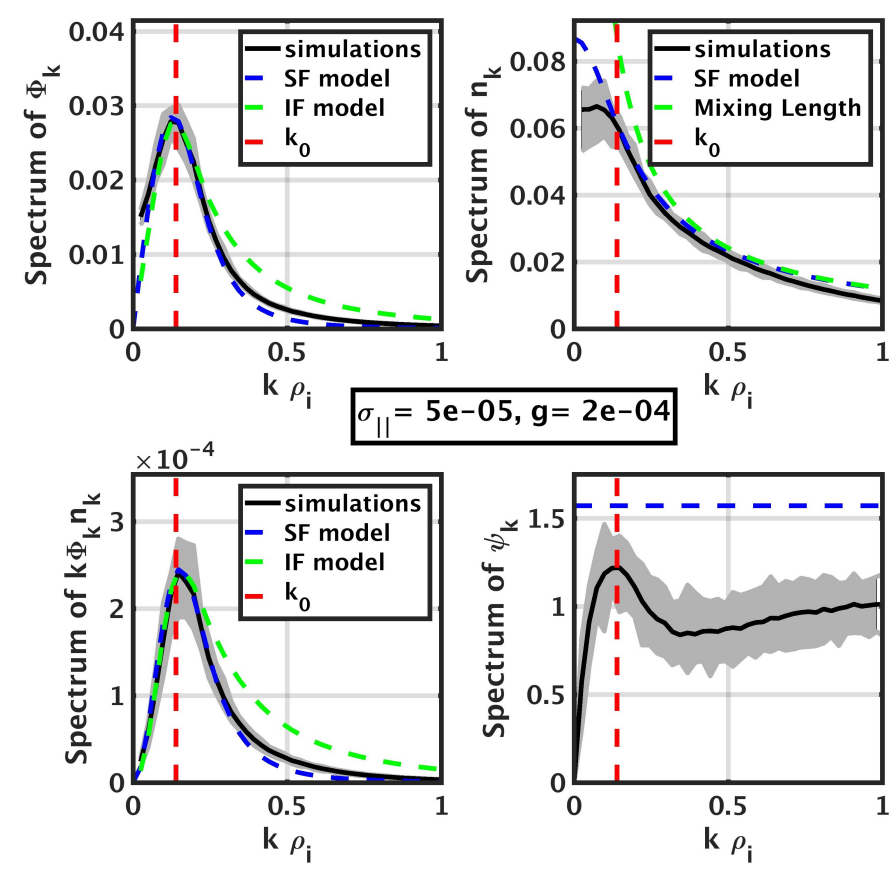

Figure 1: Time averaged poloidal spectra of potential (top left), density (top right), radial flux contribution (bottom left) and phase shift between density and potential (bottom right) from a single simulation $\left(\sigma_{\|}=5 \times 10^{-5}\right.$ and $\left.g=2 \times 10^{-4}\right)$. Grey error bars depict the extreme values of the quantity in the time range and spatial zone of study at each wavenumber value. Predictions from the isolated filament (IF) model are shown in dashed green, and dashed blue for the spectral filament (SF) model. The $\mathrm{SF}$ model prediction of the dominant wavenumber $k_{0}$ is shown as dashed red vertical lines 
As discussed previously, the SF model is able to reproduce quantitatively both density and potential spectra over a large wavenumber range. Still, the density spectrum is slightly overestimated in the low $\mathrm{k}$ domain $\left(k<k_{0}\right)$, but this has a negligible impact on global quantities as shown in the following. To verify the robustness of this agreement against the whole set of simulations, we focused the comparison on a set of relevant parameters of turbulence: the dominant wave number $k_{0}$, the amplitude of potential and density spectra at this wave number $\Phi_{k_{0}}$ and $n_{k_{0}}$, the normalized fluctuation levels of density and potential fields $\sigma_{n} / \bar{n}$ and $\sigma_{\Phi} / T_{e}$ (measured in simulations as the temporal standard deviation at fixed local positions and then averaged over these positions), and finally the density exponential decay length $\lambda$. The comparison between these transport parameters measured in the simulations and the spectral filament model predictions is shown in fig.2. Spectral properties of density and potential fluctuations as measured in the simulations are quantitatively matched by the SF model: both the value of $k_{0}$ and the spectral amplitudes at this wavenumber are predicted by the model with less than $20 \%$ error. Note that over the large range of control parameters, the dominant wavenumber varies by a factor of 2 and the spectral amplitude by about 10 . The fluctuation levels of density and potential are also well reproduced by the model. The match is quantitative for density, but the model underestimates the fluctuation level of potential by about $20 \%$, which is systematic over the set of simulations. This is still not well understood, but could be caused by the presence of zonal modes $(k=0)$ in the simulations that are not treated by the SF model. Finally, predictions for the SOL width $\lambda$ are also found to be in quantitative agreement with simulation results, over a variation range of about 3 . It shows that the model predicts well the strength of radial turbulent transport.
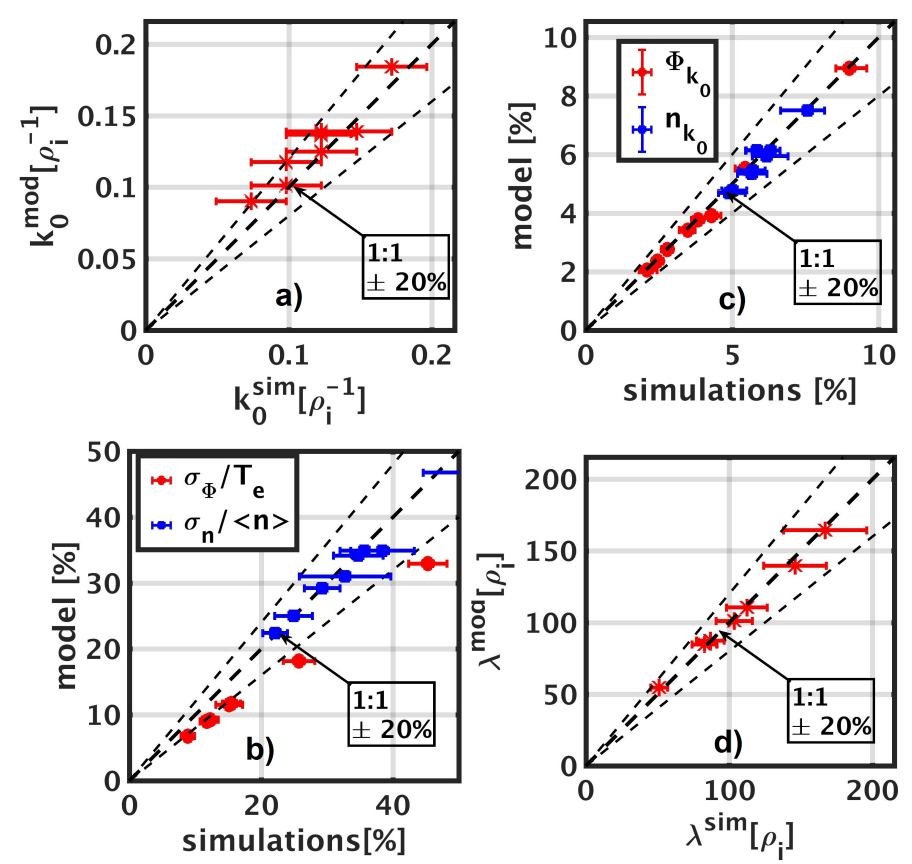

Figure 2: Comparison of the SF model predictions with TOKAM-2D simulations for a) $k_{0}$, b) the fluctuation levels of density and potential, c) properties of the mode at $k_{0}: \Phi_{k_{0}}$ and $n_{k_{0}}$, and d) density decay length $\lambda$. For simulation values, errobar bars are defined as: the spectral resolution of the simulations for $k_{0}$, extrema of time variations for $\Phi_{k_{0}}$ and $n_{k_{0}}$; and for the normalized fluctuation levels and $\lambda, 2$ times the standard deviation of their temporal and spatial fluctuations.

These quantitative agreements, covering various aspects of turbulence properties, over a large range of control parameters, establish the verification of the spectral filament model.

\section{Validation of the SF model against Tore Supra experiments}

The model addressed the description of interchange transport in simplified geometries: circular cross sections without diverted volumes, no volume source of particles in the domain of interest. Plasma discharges performed in Tore Supra can exhibit such properties [19]. Here we will focus on a set of ohmic discharges limited on the high field side of the plasma section. This series of pulses were intensively studied as part of a database for the ITER startup phase [20]. During the stationary phase of the discharges (several seconds), two reciprocating probe systems were used, both located at the top of the vacuum chamber and spanning the probe head from the wall up to the last closed flux surface. A first system holds tunnel collectors in a parallel Mach configuration, dedicated to background profile measurements [21]. The second system holds a poloidal array of small collectors, called rake probe, dedicated to fluctuation measurements [22]. The array consists in 
six protruding pins separated by $3 \mathrm{~mm}$ that are alternatively floating or biased to $-200 \mathrm{~V}$. Such system collects fluctuations of floating potential and saturation current at a frequency of $1 \mathrm{MHz}$ [7]. Unfortunately, it was not possible to coincide each tunnel probe reciprocation with a rake probe reciprocation. The tunnel probe database contains about 100 profiles collected in various discharge parameters $\left(B_{T} \in[2.6,4.1] \mathrm{T}, I_{P} \in[0.4,1.25] \mathrm{MA}\right)$, whereas the rake probe database contains about 40 fluctuation profiles collected in a similar parameter range $\left(B_{T} \in[3.1,4.1] \mathrm{T}, I_{P} \in[0.6,1.4] \mathrm{MA}\right)$.

Previous works on this database have shown that fluctuations are indeed of interchange nature, and exhibit filament like signatures in agreement with the isolated filament model [7]. It was also shown that the database of density decay length estimated by the tunnel probe could be statistically reproduced by a scaling law built from TOKAM-2D simulations [6]. These studies showed that the underlying model of interchange simulated by TOKAM-2D does represent the transport physics at play in Tore Supra ohmic circular discharges. This comparison is now extended to the whole set of experimental observable that the spectral filament model can scope with.

Applying the model to experimental conditions requires a re-normalisation of the model variables, or a normalization of the experimental set of observable. Since all relevant local plasma parameters are accessible with both probe systems, comparison of the model against Tore Supra database will be done in normalized units. In particular, the effective curvature and parallel loss rates will be expressed as $g=1.5 \rho_{i} / R$ and $\sigma \|=\rho_{i} /(\pi q R)$.

The comparison to Tore Supra database is done in two stages. First, properties of average filaments are focused. The treatment for compiling average filament properties from the reciprocating rake probe data is detailed in [7]. A database of about 7000 average filaments is obtained. Each filament is characterised by its poloidal size, radial $E \times B$ drift velocity, density perturbation normalized to background value, local electron temperature and global discharge parameters. The spectral filament model is not meant to describe individual filaments, but rather to describe the collection of filaments represented by a poloidal spectrum. Nevertheless, the model shows the existence of a dominant mode $k_{0}$ that should emerge, as an average trend, across the experimental database. This is indeed shown in fig. 3 . The experimental inverse filament size $k$ is found, in average, to be quantitatively reproduced by the prediction of $k_{0}$ (eq. 9 and 17). Note that the experimental set of average filaments exhibit a broad range of sizes that cannot be covered by the spectral filament model, obviously because the model can only predict the most probable state. A similar agreement is found for the drift velocity of the filaments, being quantitatively reproduced by $V_{k_{0}} \equiv k_{0} \Phi_{k_{0}}$, in average.

The second stage of comparison concerns transport quantities that are directly predicted by the SF model: the SOL width and fluctuation levels. In fig. 3 are shown such comparisons. The density SOL width is found to be quantitatively predicted by the model, with a deviation around unity of about $20 \%$ in average. The fluctuation levels are also well reproduced by the model, noting that the range of variation is relatively small. A striking feature of this comparison is that the model underestimates the potential fluctuation level by about $20 \%$ in average, which is similar to the mismatch found during the verification of the model against TOKAM-2D simulations.
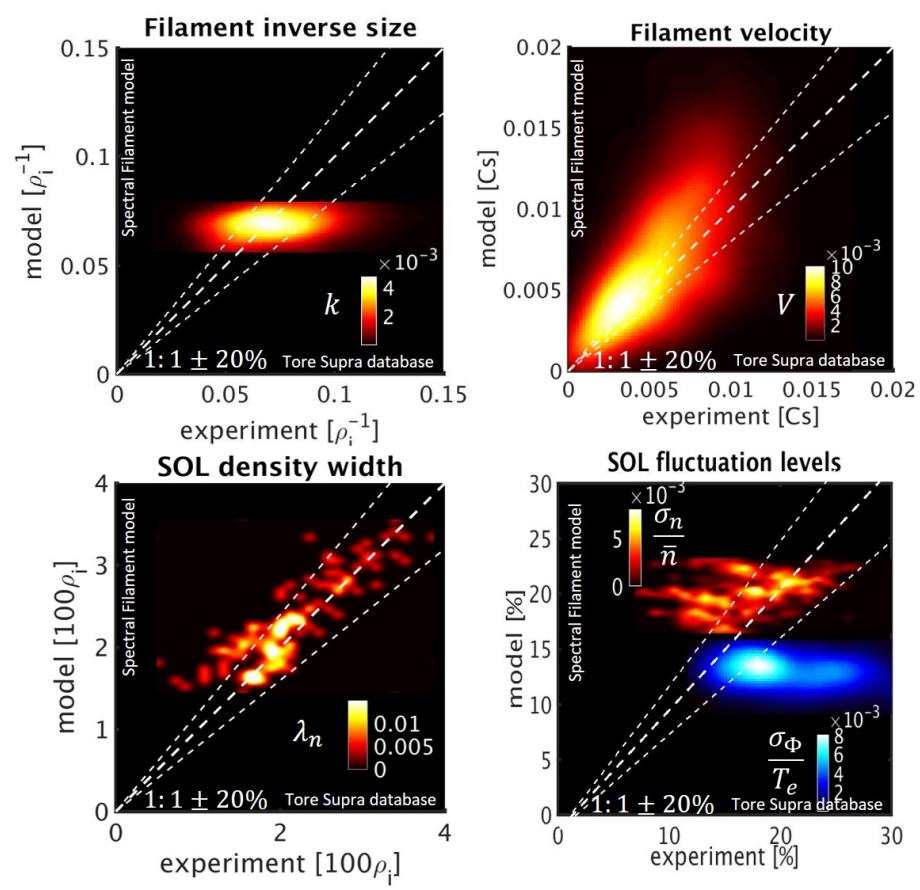

Figure 3: Maps of point concentration comparing the SF model predictions and the Tore Supra database for the dominant filament inverse size (to left), the dominant filament velocity (to right), the SOL width (bottom left) and the fluctuation levels of density and potential (bottom right) (lighter colors for higher concentration)

\section{Discussion}

\subsection{Engineering scaling laws}

In order to provide a generic application of the model in term of scaling laws based on engineering parameters, the electron and ion temperature need to be evaluated through another model. We will make the assumption that $T_{i}=T_{e}$ and use the 2 point model in 
the high collisionality regime to derive an expression of the electron temperature at separatrix:

$$
T_{e} \approx 0.13\left(\frac{q_{c y l}^{2} R P_{s e p}}{a \lambda_{q}}\right)^{2 / 7}
$$

, where $T_{e}$ is in $\mathrm{eV}, R$ and $a$ respectively the major and minor radius in meter, $P_{\text {sep }}$ the power crossing the separatrix in $\mathrm{W}$, and $\lambda_{q}$ the heat flux channel width in meter. To use this expression in the SF model, one need to estimate $\lambda_{q}$, recalling that the model predicts the density decay length $\lambda$ and not directly the heat flux decay length. To solve this, we will assume that the heat flux decay length is a constant fraction of the density decay length: $\lambda_{q}=0.5 \lambda$. This factor 0.5 is what is found in Tore Supra experiments [6], although it does not necessarily scope with evidences from diverted plasma scenarios where this ratio can be smaller and vary across different regimes (L-mode or H-mode). Anyhow, since the model is not yet addressing the diverted geometry, we follow this rough approximation for now. The model predictions are rewritten with engineering parameters only:

$$
\begin{aligned}
& \lambda_{q}[m m]=5 q_{c y l}^{0.68} B^{-0.66} R^{0.34}\left(\frac{A}{Z}\right)^{0.33} P_{\text {sep }}^{0.09} a^{-0.09} \\
& k_{0}\left[m^{-1}\right]=1.10^{3} q_{c y l}^{0.51} B^{0.74} R^{-0.26}\left(\frac{A}{Z}\right)^{-0.37} P_{\text {sep }}^{-0.1} a^{0.11}
\end{aligned}
$$

$V_{k_{0}}\left[m . s^{-1}\right]=106 q_{c y l}^{0.37} B^{-0.32} R^{-0.32}\left(\frac{A}{Z}\right)^{-0.34} P_{s e p}^{0.19} a^{-0.19}$

$$
\frac{\sigma_{n}}{\bar{n}}[\%]=33 q_{\text {cyl }}^{-0.33} B^{-0.16} R^{-0.17}\left(\frac{A}{Z}\right)^{0.08} P_{s e p}^{0.03} a^{-0.03}
$$

$$
\frac{\sigma_{\Phi}}{T_{e}}[\%]=9.25 q_{c y l}^{0.35} B^{-0.33} R^{-0.33}\left(\frac{A}{Z}\right)^{0.16} P_{\text {sep }}^{0.05} a^{-0.05}
$$

Interestingly, this scaling produced by the SF model, finds a sound agreement with multi-machine scaling laws. First, in L-mode limited [8] and diverted regimes [1] and also in $\mathrm{H}$-mode discharges [2]. Nevertheless, some discrepancies appears for (1) the non dependency on mass number in experiment and (2) the constant value which is lower in diverted plasmas and in H-mode discharges.

\subsection{Collisionality and geometry impacts on SOL width}

Halpern et al. [5, 14] proposed a similar approach in order to build a scaling law based on a linear theory around an unique wavenumber $k_{0} \rho_{i}=0.1$ and taking into account a mixing length on a mesoscale wavenumber $\sqrt{k_{0} / \lambda}$. Another difference is in the phenomenon which leads the parallel losses. In the works of Halpern et al., a plasma resistivity is invoked whereas in this study sheath resistivity is considered. Both scaling laws lead to qualitatively agreeing dependencies in the control parameters $\left(\lambda_{\text {Halpern }} \propto\right.$ $q^{0.84} B^{-0.38} R^{0.68} n^{0.07} T^{0.06} \quad$ vs $\quad \lambda_{S F}[\mathrm{~mm}]=$ $10 q^{0.55} B^{-0.73} R^{0.27}\left(\frac{A}{Z}\right)^{0.36} T_{e}^{0.36}\left(1+\frac{T_{i}}{Z T_{e}}\right)^{0.36}$. Nevertheless, some discrepancies are remarkable : (1) a dependence in electron density is not predicted by the SF model and (2) the SF model predicts an absolute value of the SOL width. The resistivity could have an important impact on the parallel dynamics in SOL in the divertor high-density regimes in which we see a broadening of the near SOL width [23]. This is addressed in more recent studies [24] based on fitting on an experimental dataset to try to quantify the turbulence contribution in transport. The SF model is able to reproduce quantitatively the SOL width and turbulent behavior in the main scrape-off layer in inner-wall limited circular plasmas. Changing to a diverted geometry has an impact on the turbulence features. This had been observed experimentally [25] and with 3D isothermal simulations [17]. Both show a variation of the SOL width of an order of magnitude. Geometrical effects or X-point impacts maybe spearheads this decrease of outgoing flux. A change in the parallel flow dynamics can also be involved in this change. A finer description of the parallel dynamics accounting for 2-pt or 3-pt model (with a 3rd point at the vicinity of the $\mathrm{X}$-point) could be used to recover diverted geometry conditions and possibly describe these last points. Moreover, the SF model offers a handling which could allow one to describe different phenomena as the turbulence in the plasma at the edge of closed flux surfaces with a resistive description of the parallel dynamics. A work on the curvature parameter $g$ has also to be done in order to describe the impact of geometrical parameters such as elongation or triangularity on the turbulence.

\subsection{Shear flows damping of the turbulence}

In previous contributions, Staebler et al. [26, 27] proposed a linear approach in order to introduce the impact of $E \times B$ shear. This point of view is comparable 
to what is done here for the expression of the potential perturbations. Indeed, they expressed the potential as the growth rate divided by the square of the wavenumber which is exactely the result obtained by the approximations made here to treat the non linear terms in the Isolated Filament model. Furthermore, this gives a promising point of view to introduce the shear effects in the model (ongoing work).

\section{Summary and conclusion}

A spectral decomposition of the filamentary transport in the scrape-off layer of tokamaks has been derived from conservation equations. Pointing a predominating wavenumber $k_{0}$ in the turbulence spectra, the model is able to predict all the features of the turbulence at this wavenumber leading to a radial flux model for the transport. This model has been verified against 2D isothermal flux driven simulations with TOKAM-2D and compared with an experimental database from inner-wall limited circular L-mode discharges in Tore Supra finding a very good quantitative agreement for (1) local properties of the turbulence i.e. wavenumber and velocities of the perturbations and (2) large-scale properties i.e. fluctuation levels and SOL width $\lambda$. This SF model leads to an expression of the SOL width $\lambda[\mathrm{mm}]=$ $10 q B^{-8 / 11} R^{3 / 11}\left(\frac{A}{Z}\right)^{4 / 11} T_{e}^{4 / 11}\left(1+\frac{T_{i}}{Z T_{e}}\right)^{4 / 11}$ which predicts values for the ITER startup conditions in again good agreement with multi-machine extrapolations $(\lambda \approx 5 \mathrm{~cm}$ according to $[8,28])$. Moreover, the dependencies of the model predictions with the plasma control parameters are close to what is observed experimentally. Aiming at validate this scaling, 3D isothermal flux driven simulations with TOKAM3X will be useful and developed in future works. Furthermore, finer effects could be included in the model such as: (1) X-point or magnetic shear, (2) density regimes and (3) shear flow damoping.

\section{Acknowledgments}

This work has been carried out within the framework of the EUROfusion Consortium and has received funding from the Euratom research and training programme 2014 - 2018 under grant agreement no. 633053 for project WP17-ER/CEA-08. The views and opinions expressed herein do not necessarily reflect those of the European Commission.

\section{References}

[1] A. Scarabosio et al, Outer target heat fluxes and power decay length scaling in L-mode plasmas at JET and AUG, J. Nucl. Mater. 438 (2013), p. S426 https://doi.org/10.1016/j.jnucmat. 2013.01.086

[2] T. Eich et al, Scaling of the tokamak near the scrapeoff layer $H$-mode power width and implications for ITER, Nuclear Fusion 53 (2013) http://dx.doi. org/10.1088/0029-5515/53/9/093031

[3] Y.Sarazin et al, Intermittent particle transport in two-dimensional edge turbulence, Physics of Plasmas, Volume 5, 12 (1998) https://doi.org/10. $1063 / 1.873157$

[4] J. Olsen et al, Scrape-off layer power fall-off length from turbulence simulations of ASDEX Upgrade L-mode,Plasma Phys. Control. Fusion 60 (2018) 085018 https://doi.org/10.1088/ 1361-6587/aace8b

[5] F.D. Halpern et al, Theory of the scrape-off layer width in inner-wall limited tokamak plasmas, Nucl. Fusion 54 (2014) 043003 http: //dx.doi .org/10. 1088/0029-5515/54/4/043003

[6] N. Fedorczak et al, Width of turbulent SOL in circular plasmas: A theoretical model validated on experiments in Tore Supra tokamak, Nuclear Materials and Energy 12 (2017) http://dx.doi.org/ $10.1016 /$ j.nme.2017.03.032

[7] N. Fedorczak et al, On the dynamics of blobs in scrape-off layer plasma: Model validation from two-dimensional simulations and experiments in Tore Supra, Contrib. Plasma Phys. (2018) http: //dx.doi.org/10.1002/ctpp.201700169

[8] J. Horacek et al, Multi-machine scaling of the main SOL parallel heat flux width in tokamak limiter plasmas, Plasma Phys. Control. Fusion 58 (2016) http://dx.doi.org/10.1088/ 0741-3335/58/7/074005

[9] J. R. Myra et al, Collisionality and magnetic geometry effects on tokamak edge turbulent transport. I. A two-region model with application to blobs, Physics of Plasmas 13, 112502 (2006)http: //dx.doi.org/10.1063/1.2364858

[10] D. A. D'Ippolito et al, Convective transport by intermittent blob-filaments: Comparison of theory and experiment, Physics of Plasmas 18, 060501 (2011)https://doi.org/10.1063/1.3594609

[11] J. R. Myra et al, Theory based scaling of edge turbulence and implications for the scrape-off layer width, Physics of Plasmas 23, 112502 (2016)http: //dx.doi.org/10.1063/1.4966564

[12] Ph. Ghendrih et al., Scaling intermittent cross-field particle flux to ITER, International Atomic Energy Agency (2004)

[13] O.E. Garcia, Stochastic Modeling of Intermittent Scrape-Off Layer Plasma Fluctuations, Physical Review Letter 108, 265001 (2012) http://dx. doi.org/10.1103/PhysRevLett.108.265001

[14] F.D. Halpern et al., A theoretical interpretation of the main scrape-off layer heat-flux width scaling for tokamak inner-wall limited plasmas, Plasma Phys. Control. Fusion 58 (2016) http://dx.doi.org/ 
$10.1088 / 0741-3335 / 58 / 8 / 084003$

[15] A.H. Nielsen et al, 2D fluid simulations of interchange turbulence with ion dynamics, 40th European Physical Society Conference on Plasma Physics (2013)

[16] F. Nespoli et al, 3D structure and dynamics of filaments in turbulence simulations of WEST diverted plasmas, Nuclear Fusion 59 (2019)https: //doi.org/10.1088/1741-4326/ab2813

[17] P. Tamain et al., The TOKAM3X code for edge turbulence fluid simulations of tokamak plasmas in versatile magnetic geometries J. Comput. Phys 321 (2016) 606623 http://dx .doi.org/10.1016/ j.jcp. 2016.05.038

[18] S.I. Krasheninnikov, On scrape off layer plasma transport, Physics Letters A 283 (2001)

[19] N. Fedorczak et al, Electrostatic transport in L-mode scrape-off layer plasmas in the Tore Supra tokamak. I. Particle balance, physics of plasmas 19 (2012) http://dx.doi.org/10.1063/1.4739058

[20] J.P. Gunn, Scrape-off layer power flux measurements in the Tore Supra tokamak, Journal of Nuclear Materials 438 S184-S188 (2013) https://doi.org/ 10.1016/j . jnucmat.2013.01.055

[21] J.P. Gunn, Electric Probes in Tokamaks: Experience in Tore Supra, Contrib. Plasma Phys 51 (2011) https://doi.org/10.1002/ctpp. 201000074

[22] N. Fedorczak et al, Electrostatic transport in L-mode scrape-off layer plasmas of Tore Supra tokamak.
II. Transport by fluctuations, physics of plasmas 19 (2012) https://aip.scitation.org/doi/10. 1063/1.4739059

[23] Carralero et al., A study on the density shoulder formation in the SOL of H-mode plasmas, Nuclear Material and Energy 12 (2017) http://dx.doi. org/10.1016/j.nme.2016.11.016

[24] T. Eich et al., Turbulence driven widening of the near-SOL power width in ASDEX Upgrade $H$ Mode discharges, Nuclear Fusion 60 (2020) https: //doi.org/10.1088/1741-4326/ab7a66

[25] D.L. Rudakov et al., SOL width in limited versus diverted discharges in DIII-D Journal of Nuclear Materials 415 (2011) S387S390 http://dx.doi. org/10.1016/j.jnucmat.2010.10.036

[26] G.M. Staebler et al., New Paradigm for Suppression of Gyrokinetic Turbulence by Velocity Shear Physical Review Letter 110 (2013) 055003 http: //dx. doi.org/10.1103/PhysRevLett.110.055003

[27] G.M. Staebler et al., A new paradigm for $E$ B velocity shear suppression of gyro-kinetic turbulence and the momentum pinch Nuclear Fusion (2013) 113017 http://dx.doi.org/10.1088/ 0029-5515/53/11/113017

[28] K. McCormick et al., ITER edge database investigations of the SOL width Journal of Nuclear Materials 266-269 (1999) 99-108 https://doi .org/10. 1016/S0022-3115(98)00521-2 